\title{
Combination of Structure-Performance and Shape- \\ Performance Relationships for Better Biphasic Release in \\ Electrospun Janus Fibers
}

5 Xiaolu Zheng ${ }^{1}$, Shixiong Kang ${ }^{1}$, Ke Wang ${ }^{1}$, Yaoyao Yang ${ }^{1}$, Deng-Guang $\mathrm{Yu}^{1 *}$, Fuxian $\mathrm{Wan}^{2}$, Gareth R. Williams ${ }^{3 *}$, Sim-Wan Annie Bligh ${ }^{4 *}$

${ }^{1}$ School of Materials Science \& Engineering, University of Shanghai for Science \& Technology, Shanghai 200093, China.

$10{ }^{2}$ College of Chemistry and Material Science, Shandong Agricultural University, Tai'an 271018, China

${ }^{3}$ UCL School of Pharmacy, University College London, 29-39 Brunswick Square, London WC1N 1AX, UK

${ }^{4}$ School of Health Sciences, Caritas Institute of Higher Education, 2 Chui Ling Lane,

15 Tseung Kwan O, New Territories, Hong Kong 999077, China

20 Corresponding Authors:

Prof. Deng-Guang Yu

School of Materials Science \& Engineering

University of Shanghai for Science and Technology

Address: 516 Jungong Road, Yangpu District, Shanghai 200093, China 
25 E-mail: ydg017@usst.edu.cn

Tel/Fax: +86-21-55270632

*E-mail: g.williams@ucl.ac.uk (GRW)

*Email: abligh@cihe.edu.hk (SWAB)

30

\section{Abstract}

In nature, the combination of composition, structure, and shape determines the matter's functional performance to a large extent. Inspired by which, two electrospun Janus nanofiber formulations were created using side-by-side electrospinning in this work.

40 Tamoxifen citrate (TAM) was used as a model drug and ethyl cellulose (EC) and polyvinylpyrrolidone K60 (PVP) as the polymer carrier matrices. The fibers have linear cylindrical morphologies and distinct Janus structures by scanning electron microscopy. One side of the fibers took a round shape, while the other was crescent-shaped. The drug was present in both polymer matrices in the form of amorphous solid dispersions, owing

45 to strong intermolecular interactions between drug and polymer. In vitro dissolution tests demonstrated that both sets of fibers could provide biphasic drug release due to the difference in solubility of PVP and EC. The different shape of TAM-EC and TAM-PVP side of the Janus structure resulted in a considerable variation in the drug release profiles. 
The Janus structure with crescent TAM-PVP side and round TAM-EC side gave a more

50 rapid burst release in the first phase of release, and slower sustained release in the second phase. This work thus reports a new strategy for systematically developing advanced functional nanomaterials based on both shape- and structure-performance relationships.

\section{Keywords}

Side-by-side electrospinning; Janus nanostructure; shape-performance relationship; side 55 allocation; biphasic release

\section{Chemical compounds studied in this article:}

Tamoxifen citrate (PubChem CID: 2733525);

60 Polyvinylpyrrolidone (PubChem CID: 6917);

Ethyl cellulose (PubChem CID: 24832091);

Dichloromethane (PubChem CID: 6344);

Ethanol (PubChem CID: 702);

Basic fuchsin (PubChem CID: 3081760);

65 Methylene blue (PubChem CID: 6099);

Sodium hydroxide (PubChem CID: 14798). 


\section{Introduction}

In addition to the components of a substance, its internal structure and outer shape are essential factors in determining its functional properties. Novel nanostructures introduced into the manufacturing of advanced materials enable new science and technology (Isaacoff and Brown, 2017; Chen, et al., 2019). During the past several decades, numerous efforts have been devoted to the creation of nanostructures for

75 developing functional products. In comparison, minimal attention has been paid to the external shapes of nanomaterials, which plays a vital role in functional performance (Mitragotri and Lahann, 2009). Further, few works can be found on developing functional nanomaterials by controlling both inner structure and outer shape. However, Emerging Janus structures consist of two different regions that can be individually

80 designed in terms of both chemical composition and functionality and linked together and behave as one entity (Han, et al., 2018; Song, et al., 2018). Two compartments of the Janus structure can directly interact with the exterior environment compared to other twopart systems, which enables Janus-structured materials to show even more extraordinary function in various kinds of applications.

Electrospinning is a simple and effective top-down, one-step method to produce polymer-based nanofibers (Nagy, et al., 2015), particularly polymer-based nanostructures (Wang, et al., 2020a; Jiang, et al., 2017; Liao, et al., 2017). While the majority of studies in the literature employ the simplest uni-axial setup (using one polymer solution to prepare monolithic fibers), it is popular and thrilling to process 
multiple fluids simultaneously. In recent years, researchers have invested considerable time and effort in the generation of complex nanostructures using a macro-structured spinneret as a template to dispense multiple fluids in an organized manner (Zhu, et al., 2019; Agarwal, et al., 2008) except the free-surface electrospinning (Bai, et al., 2021; Kang, et al., 2021). Besides, superior mechanical and thermal properties of fibers were

95 invested by researchers (Jiang, et al., 2020; Yao, et al., 2019). It was even found that electrospinning can be used to provide superior structure-dependence of polymer fibers on uni-axial tensile properties overcoming great conflict between strength and toughness (Liao, et al., 2019). The multifluid electrospinning processes reported to date including coaxial electrospinning (Moghe and Gupta, 2008), tri-axial electrospinning (Hou, et al., 100 2020; Wang, et al., 2020b), modified coaxial/triaxial electrospinning (Ding, et al., 2020), and side-by-side electrospinning (Zhao, et al., 2021). Using above-mentioned advanced processes, fiber diameter, composition, shape, morphology, structure, and dimensionality (form 1D to 2D and 3D) can be controlled by adjusting both the solution parameters (e.g., polymer molecular weight and concentration) and processing 105 parameters (voltage, flow rate, and distance between the spinneret and collector) (Yoon, et al., 2018; Zhang, et al., 2019; Duan and Greiner, 2019).

Nanomedicine is a rapidly evolving form of therapy that holds great promise in drug delivery and has been remarkably and widely explored in cancer treatments. Everincreasing efforts are being devoted to designing complex nanostructures for application 110 in new drug delivery systems (DDSs) (Chang, et al., 2020; Labbaf, et al., 2014; Zheng, 
et al., 2020; Ramzy, et al., 2017). Myriad drug-loaded functional nanomaterials that can provide precision and personalized therapies have been reported (Ibrahim, et al., 2020; Santos, et al., 2018). Thus, nanostructured medicines are gaining more and more clinical attention, which is because the nanostructure of the carrier provides new properties and can be more potent for targeted therapy of tumors (Kim, et al., 2010). The controlled release of anti-cancer drugs using nanostructures is expected to provide further clinical developments when components, inner structure, and outer shape of the nanomedicine are systematically considered. The physicochemical properties of excipients or carrier materials (such as lipids and polymers) are frequently exploited to control the drug 120 release profile (Chen, et al., 2018; Viviane, et al., 2017; Yu, 2021). For example, watersoluble polymers such as polyvinylpyrrolidone (PVP) can be used to develop immediate drug delivery systems. In contrast, water-insoluble polymers such as cellulose acetate (CA), can provide sustained release (Aidana et al., 2021). Such work has increasingly been performed at the nanoscale to generate new advanced DDSs (Démuth, et al., 2015;

125 Grossen, et al., 2017; Li, et al., 2020). However, the influence of the outer shape of nanostructure is carelessly overlooked, in part because few techniques can be employed to manipulate the outer shape at the nanoscale, and fewer can systematically tailor the composition, internal structure, and outer shape. The pharmaceutical properties of Janus fibers from electrospinning have not been widely explored to date, although their 130 potential is prominent. Recently, a hydrophilic Janus nanocomposite DDS for the fast release of orally administered water-insoluble drugs have proven the very potential (Wang, et al., 2018). Still, exploiting Janus structures to prepare complex nanomaterials 
and accomplish improved functional performance still holds new promises for applied and functional materials.

In this work, a new strategy for developing next-generation functional nanomaterials using side-by-side electrospinning for which two fluids are ejected from adjacent parallel nozzles is reported, systematically considering composition, structure, and shape. Tamoxifen (TAM), an adjuvant chemotherapeutic drug used in the treatment of breast cancer (Stearns, 2018; Burstein, et al., 2016; Jordan, 2017), was selected as a model drug.

140 Precision medicine is highly desired for TAM, to ensure that it quickly reaches a therapeutic systemic concentration, which is later to maintain for a relatively long period (Webster, 2006). Two sets of Janus fibers were prepared with different composition allocations in Janus structure. The first consisted of a round TAM-EC side and a crescent TAM-PVP side, while the second comprised a round TAM-PVP side and a crescent

145 TAM-EC side. The fibers were characterized in detail, and the difference in drug release profiles were measured to disclose the combination effect of structure-performance and shape-performance relationships.

\section{Experimental section}

\subsection{Materials}

Tamoxifen citrate was purchased from Sunary Pharmaceutical Corp. (Suzhou, China). Polyvinylpyrrolidone K60 (PVP K60, $\left.M_{\mathrm{w}}=360,000\right)$ and ethyl cellulose (EC) were obtained from Sigma-Aldrich Corp. (Shanghai, China) and the Aladdin Chemistry Co., Ltd. (Shanghai, China), respectively. Analytical grade dichloromethane (DCM) and 
anhydrous ethanol were bought from the Sinopharm Chemical Reagent Co., Ltd.

155 (Shanghai, China). All other chemicals were analytical grade, and water was double distilled before use.

\subsection{Electrospinning}

Two solutions were prepared for electrospinning. The TAM-PVP solution consisted of $8 \%(w / v)$ PVP K60 and 2\% (w/v) TAM in a mixture of ethanol and DCM with a volume ratio of 1:1. The TAM-EC solution comprised 28\% (w/v) EC and 3\% (w/v) TAM in a mixture of ethanol and DCM at 1:1 (v/v). For optimizing the experimental conditions, two dyes, basic fuchsin and methylene blue, were added into the PVP-TAM side and ECTAM side, respectively, at a concentration of $1 \times 10^{-3} \mathrm{mg} / \mathrm{mL}$.

Building on progress reported in previous studies (Wang, et al., 2018; Wang, et al.,

165 2020c), a homemade eccentric spinneret was developed for implementing side-by-side electrospinning. Two syringe pumps (KDS100 and KDS200, Cole-Parmer, Vernon Hills, 1L, USA) were employed to drive the working fluids. A power supply (ZGF 60KVZ, Sute Corp., Shanghai, China) provided an electric field. A digital camera (PowerShot A490, Canon, Tokyo, Japan) was used to capture the working processes. Four kinds of

170 fibers were electrospun. The monolithic F1 and F2 formulations were collected at a flow rate of $1.0 \mathrm{~mL} / \mathrm{h}$, while the Janus fibers $\mathrm{F} 3$ and $\mathrm{F} 4$ were fabricated with a flow rate of 1.0 $\mathrm{mL} / \mathrm{h}$ for both sides. The voltages lay between 12 to $15 \mathrm{kV}$.

\subsection{Morphology}


Scanning electron microscopy (SEM; Quanta 450 FEG instrument, FEI, Hillsboro,

175 OR, USA) was used for evaluating the nanofibers' morphology. Before SEM study, the samples were coated with a gold sputter. Transmission electron microscopy (TEM; Hitachi H-800 instrument, Tokyo, Japan) was used to probe the internal structure of F3 and F4.

\subsection{Physical Status}

180

X-ray diffraction (XRD, D/Max-BR, Rigaku, Japan) was carried out over the $2 \theta$ range from $10^{\circ}$ to $60^{\circ}$. The diffractometer was supplied with $\mathrm{Cu} \mathrm{K} \alpha$ radiation at $40 \mathrm{kV}$ and $30 \mathrm{~mA}$. Attenuated total reflectance Fourier transform infrared (ATR-FTIR) spectra were collected on a Nicolet IS10 Instrument (Madison, WI, USA). Experiments were performed in the range $500-4000 \mathrm{~cm}^{-1}$ with a resolution of $2 \mathrm{~cm}^{-1}$. Differential scanning

185 calorimetry (DSC; Q200 calorimeter, TA Instruments, Billerica, MA, USA) was performed with $10 \mathrm{mg}$ of each sample being heated from 0 to $250{ }^{\circ} \mathrm{C}$ at a rate of $10^{\circ} \mathrm{C} / \mathrm{min}$ under a purge of nitrogen gas at a flow rate of $10 \mathrm{~mL} / \mathrm{min}$.

\subsection{In vitro dissolution tests}

Drug release properties were conducted according to the Chinese Pharmacopoeia

190 (2015 EDN) Method II. A paddle method was employed using a constant temperature oscillating incubator apparatus (THZ312, Jinghong Instrument Co., Ltd., Shanghai, China). $200 \mathrm{mg}$ of TAM-loaded nanofibers were placed into $600 \mathrm{ml}$ of $0.01 \mathrm{~N} \mathrm{HCl}$ for 1 $\mathrm{h}$, and later $0.24 \mathrm{~g}$ sodium hydroxide was added to the dissolution medium to adjust its $\mathrm{pH}$ value to 7.0. All experiments were undertaken at a fixed temperature of $37 \pm 1^{\circ} \mathrm{C}$, 
with stirring at $60 \mathrm{rpm}$ and under sink conditions of $\mathrm{C}<0.2 \mathrm{C}_{\mathrm{s}}$. At constant time points, $5.0 \mathrm{~mL}$ of the release medium was withdrawn from the dissolution medium and replaced with fresh pre-heated medium corresponding to the dissolution medium at different time points to maintain a constant volume. After filtration through a $0.22-\mu \mathrm{m}$ filter, all samples were analyzed at $\lambda_{\max }=278 \mathrm{~nm}$ using a UV/vis spectrophotometer (UV-1101II, Tianmei

200 Instrument Co., Ltd, Shanghai, China). Experiments were carried out six times, and the actual cumulative percentage of drug released from the electrospun fibers was calculated using a predetermined calibration curve (Equation 1) (Wang, et al., 2020c).

$$
P \%=\frac{\rho_{n} \times V_{0}+\sum_{i=1}^{n-1} \rho_{i} \times V}{Q_{0}} \times 100
$$

where $\mathrm{V}_{0}$ refers to the volume of the dissolution medium $(600 \mathrm{~mL}), \mathrm{V}$ is the volume 205 of the withdrawn sample $(5 \mathrm{~mL}), \mathrm{Q}_{0}$ is the theoretical amount of TAM in each fiber $(\mathrm{mg})$, $\rho_{\mathrm{n}}$ is the drug concentration determined in the nth aliquot $\left(\mathrm{mg} \mathrm{L}^{-1}\right)$, and $\rho_{\mathrm{i}}$ is the drug concentration in the ith aliquot $\left(\mathrm{mg} \mathrm{L}^{-1}\right)$.

\section{Results and Discussion}

\subsection{Side-by-side electrospinning and the eccentric spinneret}

A diagram of a typical side-by-side electrospinning system is presented in Fig. 1a. The system can be employed to carry out both single and side-by-side electrospinning. It consists of four key components: high-voltage power supply, two syringe pumps, a collector, and a spinneret (Li, et al., 2017). A homemade spinneret was employed here to provide a template for Janus fibers producing. While in the coaxial process, the key 
interactions are those between the electric field force and the charged fluid (Du, et al., 2018; Cheng, et al., 2019), in the side-by-side process, the electric field force has a significant effect on both fluids simultaneously, which makes the implementation of sideby-side electrospinning more challenging than the coaxial process.

Before producing Janus fibers, monolithic fibers consisting of PVP(TAM) (F1) and

$220 \mathrm{EC}(\mathrm{TAM})(\mathrm{F} 2)$ were prepared. The monolithic fibers were undertaken using the same spinneret with one of the syringe pumps being turned off. To estimate the effect of side allocation for final fiber-forming, two types of Janus fibers, F3 and F4, were then prepared (Table 1).

Traditionally, the spinneret consisting of two parallel capillaries has been used to 225 produce Janus fibers. However, this kind of spinneret often fails to create integrated Janus structures. When the two working fluids are extruded from the nozzle, they bear the same amount of electric charges, which inevitably results in electric repulsion between them. Combined with their small contact area, they begin to separate from each other. It is believed to be the main reason that the publications of Janus fibers are 230 substantially fewer than those of core-sheath fibers.

The spinneret (Figs. 1b and 1c) used in this work is made up of three needles, which are $32 \mathrm{G}$ (with outer/inner diameters of $0.28 / 0.11 \mathrm{~mm}), 21 \mathrm{G}(0.81 / 0.51 \mathrm{~mm})$, and $16 \mathrm{G}$ $(1.51 / 1.19 \mathrm{~mm})$. Compared to a parallel-capillary spinneret, this arrangement has three advantages: 1) its nozzle provides a continuous and full charged surface around the outer 235 metal capillary, which is useful for eliminating the repulsive forces between two fluids; 
2) two working fluids have an enlarged contact area, which helps to prevent fluid separation; 3 ) the round nozzle is slightly beyond the crescent nozzle (by about $0.2 \mathrm{~mm}$; Fig. 1d), which is helpful for preventing possible diffusion between two working fluids. In contrast, in the parallel-capillary spinneret, the two nozzles must be at the same length; 240 otherwise, the repulsion between the two fluids will be increased.

Fig. 1. Electrospinning system. (a) A schematic of the electrospinning system for producing nanofibers F1 to F4. (b) Sketch map about the inner structure of homemade spinneret. (c) The overview digital photo of the spinneret. (d) The digital photo of the 245 spinneret nozzle.

Four different sorts of nanofibers, including two types of monolithic fibers, namely F1 (TAM-PVP) and F2 (TAM-EC) and two types of Janus fibers, namely F3 (round TAM-EC side, crescent TAM-PVP side) and F4 (round TAM-PVP side, crescent TAM-

250 EC side) (Table 1), were prepared using the homemade apparatus (Fig. 1b). Shown in Fig. 1c, the syringe containing the side fluid for forming the round side (TAM-EC in F3) was directly inserted into the eccentric spinneret, and was fixed on the syringe pump. The other for forming the crescent side (TAM-PVP in F3) was guided to the eccentric spinneret through a silica tubing. The high voltage was conveyed to the spinneret through 255 an alligator clip. An aluminum foil was grounded to collect the nanofibers. After the completion of the installation, the whole system was ready to conduct electrospinning process. The high-voltage power supply could be turned on and gradually increased until 
fibers formed. Digital pictures of the working processes and different Taylor cones can be observed through a camera at a $12 \times$ magnification.

260 Table 1 Details the electrospinning processes

\begin{tabular}{llllll}
\hline \multirow{2}{*}{ No. } & Process & \multicolumn{2}{l}{ Composition } & \multicolumn{2}{l}{ Flow rate $(\mathrm{mL} / \mathrm{h})$} \\
\cline { 3 - 6 } & & Round $^{\mathrm{a}}$ & Crescent $^{\mathrm{b}}$ & Round $^{\mathrm{a}}$ & Crescent $^{\mathrm{b}}$ \\
\hline F1 & Single & -- & PVP+TAM & -- & 1.0 \\
F2 & Single & EC+TAM & -- & 1.0 & -- \\
F3 & Side-by-side & EC+TAM & PVP +TAM & 1.0 & 1.0 \\
F4 & Side-by-side & PVP+TAM & EC+TAM & 1.0 & 1.0 \\
\hline
\end{tabular}

a This solution is made up of $8 \%(\mathrm{w} / \mathrm{v})$ PVP K60 and 2\% (w/v) TAM in a mixture of ethanol and dichloromethane $(1: 1, \mathrm{v} / \mathrm{v})$.

$\mathrm{b}$ This solution comprises $28 \% \mathrm{w} / \mathrm{v}$ EC and $3 \% \mathrm{w} / \mathrm{v}$ TAM in a mixture of ethanol and dichloromethane $(1: 1, \mathrm{v} / \mathrm{v})$.

The electrospinning system is shown in Fig. 2a, and Fig. 2b gives an enlarged image showing the connection of the spinneret with the working fluids and power supply. When preparing the monolithic fibers $\mathrm{F} 1$, the pump connected to round nozzle was switched off, as exhibited in Fig. 2c. The resultant electrospinning process involved a typical

270 Taylor cone, straight fluid jet, and an unstably bending and whipping region. The Taylor cone had a red color due to the presence of a small amount of basic fuchsin $\left(1 \times 10^{-3}\right.$ $\mathrm{mg} / \mathrm{mL}$ ) in the solution for clear observation. A typical process for generating the ECTAM fibers F2 with the pump connected to crescent nozzle being turned off is shown in Fig. 2d. Here methylene blue $\left(1 \times 10^{-3} \mathrm{mg} / \mathrm{mL}\right)$ was added to aid visualization of the 
process. Still, a Taylor cone was seen, followed by a straight fluid jet and an unstable region, and the whole process terminated with the deposition of solid fibers on the collector. In this case, however, semi-solid substances were seen to be formed around the nozzle, resulting in frequent clogging of the spinneret.

When the two side fluids were extruded from side-by-side nozzles simultaneously 280 for preparing Janus fibers F3, a typical side-by-side electrospinning process could be observed in Fig. 2e. A compound Taylor cone was exhibited in the bottom inset of Fig. $2 \mathrm{e}$ with the inclusion of dyes permitting easy visualization. The two side fluids were swapped to prepare the Janus fibers F4 (Table 1 and Fig. S1 in the Supporting Information). While PVP-TAM solution could be easily spun independently, clogging of the spinneret was seen when the EC-TAM solution was extruded only from the crescentshaped nozzle.

Fig. 2. Implementation of the electrospinning: (a) The homemade electrospinning system. (b) The connection of the spinneret with the working fluids and power supply. (c) Single290 fluid electrospinning for preparing F1 using the crescent-shaped nozzle. (d) Single-fluid electrospinning of F2 using the round nozzle. (e) Side-by-side spinning for creating the Janus F3 nanofibers.

The clogging of the nozzle is a frequent phenomenon in electrospinning, particularly 295 when solutions containing natural polymers in volatile solvents utilized. In this study, the EC-TAM solution clogged both the round and crescent nozzle of the spinneret when it was subjected to single-fluid electrospinning process (Fig. S2 in the Supporting 
Information). Manual removal of the semi-solid blocks on the spinneret was required to generate monolithic EC-TAM nanofibers. However, when the EC-TAM solution was

300 processed simultaneously with PVP-TAM using the eccentric spinneret, no blocking of the spinneret was noted. The side-by-side electrospinning process was always robust and ran continuously. The PVP-TAM solution thus played an essential role in ensuring the smooth preparation of fibers F3 and F4.

\subsection{Morphology and Janus structure}

The scanning electron microscopy (SEM) images in Figs. 3a to 3d reveal that all the fibers have a linear shape and smooth surfaces. All four sets of fibers, including the fibers F1 composed of PVP and TAM (Fig. 3a) and generated from crescent nozzle, are cylindrical. It is to be expected that the fibers F2 composed of EC and TAM (Fig. 3b) and generated from the round nozzle have a cylindrical shape (Fig. S3b in the Supporting

310 Information). Similarly, it is anticipated for F3 (Fig. 3c) and F4 (Fig. 3d), both of which were produced using the whole spinneret. It suggests that the shape of the spinneret nozzles have little influence on the shape of the resultant nanofibers during single-fluid electrospinning process. When the working fluids are dispensed from the crescentshaped nozzle, the fluid will quickly change its crescent shape to a cylindrical shape to

315 form the initial Taylor cone, as a result of surface tension and surface charge repulsion.

The Image J software was employed to estimate the average diameter and size distribution of the fibers (over 100 different measurements in SEM images). The results showed that fibers F1, F2, F3, and F4 had an average diameter of $336 \pm 56 \mathrm{~nm}, 324 \pm 73$ 
$\mathrm{nm}, 579 \pm 77 \mathrm{~nm}$, and $816 \pm 113 \mathrm{~nm}$, respectively. It is obvious that, despite quite little

320 influence on shape of monolithic fibers from spinneret nozzles, EC as a polymer matrix has a relatively smaller average diameter than PVP. And EC solution had a much higher polymer concentration, when in side-by-side electrospinning, the diameter could be shifted under different solvent evaporation rates caused by surface area in Taylor cone. When evaporation rate is high enabled by larger surface area, retarding the exhaustion of

325 the solvent in the middle of the fluid jets and creating a relatively low-density internal structure and bigger diameter. F4 that PVP in round nozzle and EC in crescent nozzle had a much bigger average diameter than F3 that EC in round nozzle and PVP in crescent nozzle for the larger cross-sectional area of crescent nozzle. After exchanging nozzles, monolithic nanofibers consisting of PVP-TAM and EC-TAM prepared from the round 330 and crescent nozzle had an estimated diameter of $319 \pm 58 \mathrm{~nm}$ and $315 \pm 67 \mathrm{~nm}$, respectively. SEM images are shown in Fig. S3 in the Supporting Information.

TEM images of F3 and F4 are presented in Fig. 3. Both formulations have a clear Janus structure, with the crescent side having a lighter contrast than the round side. The round side of EC-TAM in fibers F3 is approximately $420 \mathrm{~nm}$ in size, and the crescent

335 PVP-TAM side around $158 \mathrm{~nm}$. For fibers F4, the PVP-TAM round size is about 559 $\mathrm{nm}$, and the crescent EC-TAM side is ca. $257 \mathrm{~nm}$. The bright field in TEM images results from three aspects: composition with high molecular weight, high density, and thickness. The reasons for the crescent side always having a lighter gray shade than the round side when observed is expected to be that it had a smaller thickness and also likely a reduced 
density owing to rapid evaporation of solvent because of the larger surface area on this side of the Taylor cone.

Fig. 3. SEM images of fibers F1 to F4 and their diameter distributions: (a) F1; (b) F2; (c) F3; and (d) F4; TEM images of (e) F3; (f) F4.

\subsection{Physical state and compatibility}

In the X-ray diffraction (XRD) patterns, raw TAM shows sharp Bragg reflections owing to the crystalline nature of the pure drug. In contrast, XRD patterns of PVP and EC both exhibit a diffuse background with two diffraction halos, indicating that both are amorphous polymers (Fig. 4a). It is clear that all the drug-loaded fibers, whether from single-fluid electrospinning (F1 and F2) or side-by-side electrospinning (F3 and F4), display no Bragg reflections in their patterns, but rather broad halos. TAM has thus been imbedding into monolithic (F1 and F2) and Janus nanofibers (F3 and F4) in the form of an amorphous solid dispersion.

Fig. 4. Physical state and compatibility data: (a) XRD patterns; (b) DSC curves (exo up); and (c) FTIR spectra and molecular structures. 
DSC data for the raw materials and fibers are presented in Fig. 4b. The crystalline TAM shows a sharp peak at $148.2{ }^{\circ} \mathrm{C}$, corresponding to its melting point. The two polymers, PVP K60 and EC, exhibit no melting events. PVP K60 has a broad 365 endothermic peak resulted from dehydration at between ca. 50 and $150{ }^{\circ} \mathrm{C}$, in addition to glass transitions at $98.5^{\circ} \mathrm{C}$. None of the fibers shows any melting endotherm, indicating that TAM was fully converted into amorphous nanocomposites. Only water loss endotherm and glass transition temperatures can be seen in the traces. These results concur with those from XRD.

370 To probe the stability of the nanofibers, it is vital to explore the compatibility between the drug and the polymer matrices. FTIR spectra were thus collected to examine possible secondary interactions between the fiber components, and are shown in Fig. 4c. The raw materials TAM, EC, and PVP have characteristic peaks at $1740 \mathrm{~cm}^{-1}$ (stretching of $-\mathrm{C}=\mathrm{O}$ ), $1063 \mathrm{~cm}^{-1}$ (stretching vibration of $-\mathrm{O}-$ of $\left.\mathrm{C}-\mathrm{O}-\mathrm{C}\right)$, and $1662 \mathrm{~cm}^{-1}(-\mathrm{C}=\mathrm{O}$

375 stretching), respectively. However, no characteristic peak from TAM at $1740 \mathrm{~cm}^{-1} \mathrm{can}$ be seen in the spectra in any of the fibers. This phenomenon indicates that TAM molecules have formed hydrogen bonds with PVP or EC molecules. There are both -OH and $-\mathrm{C}=\mathrm{O}$ groups in a TAM molecule. Thus, it can act as both an electron acceptor and a donor for forming hydrogen bonds (Fig. 4c). Besides, other secondary interactions such 380 as hydrophobic interactions (e.g., between the benzene rings in TAM with the pyrrole rings in PVP or alkyl groups in EC) can enhance drug-polymer compatibility, and thus the physical stability of the nanofibers. 


\subsection{In vitro drug release profiles}

To determine which side of the Janus structure dissolves first, a simple dissolution experiment was conducted. The Janus fibers were briefly placed into water, and then the residual sections were dried and sampled for SEM analysis. They were verified to be cylindrical in the case of F3 (Fig. 5a) and flat of F4 (Fig. 5b). Dissolution experiments were also carried out on Janus nanofibers produced from the dye-loaded solutions. Which side of the Janus structure dissolves first can be determined according to the color of the

390 dissolution medium after removing the residual fiber mats. As shown in Fig. S4 in the Supporting Information, the TAM-PVP side of the fibers (containing the red dye basic fuchsin) always dissolved first, making the dissolution media have a slightly red color, regardless of whether it located in the round or crescent side of the Janus fiber. It is as anticipated owing to the high solubility of PVP.

Fig. 5. SEM images of the residual sections of the fiber mats after the dissolution of the PVP compartment: (a) F3; (b) F4; In vitro drug release and the shape-action relationship: (c) In vitro dissolution profiles of full-time range with an inset picture about the first 60min. (d) Comparisons between F3 and F4 about the specific percentage points. (e) A 400 diagram showing the shape-action relationship for manipulating drug sustained-release behaviors.

Dissolution test results are exhibited in Figs. 5c to 5e. As would be anticipated, the fibers F1 composed of PVP and TAM showed a very rapid burst release, with $98.5 \pm 1.5 \%$ of the loaded drug being freed into the dissolution media within $1 \mathrm{~h}$. In contrast, F2 composed of TAM and EC showed sustained release profile. After 1h, $31.6 \pm 5.7 \%$ of 
the TAM payload was released, with $99.8 \pm 5.6 \%$ TAM being freed into dissolution media after 48 h (Fig. 5c).

The Janus nanofibers F3 and F4 both showed biphasic drug release profiles. As 410 shown in the inset of Fig. 5c, the release percentages for F3 and F4 in the first 1 minute were $38.1 \pm 6.3 \%$ and $33.4 \pm 4.5 \%$, respectively. The theoretical drug content in the PVPTAM side was $40 \%$ of the total drug payload. Approximately 2.0 and 5.0 minutes were needed to release $40 \%$ of drug content for Janus fibers F3 and F4, respectively (Fig. 5d). Thus, the F3 system showed a faster initial release than F4. The crescent shape of the 415 PVP-TAM side in F3 therefore offered advantages over the round shape in F4. The crescent shape had both a larger surface area and a reduced thickness, making it easier for the PVP to absorb water, swell, disentangle, and to dissolve into water, thereby freeing the incorporated TAM molecules.

After one hour of dissolution, $49.8 \pm 5.8 \%$ and $59.3 \pm 5.7 \%$ of the loaded drug was 420 released from F3 and F4 (inset of Fig. 5c). During the electrospinning processes, the diffusion of drug molecules from one side to the other side can be neglected owing to the very rapid drying process of electrospinning. Given that $40 \%$ of the drug was loaded in the PVP side of the fibers, which indicated that $9.8 \%(49.8 \%-40 \%)$ of the loaded TAM was released from the round EC-TAM side of fibers F3, and $19.3 \%(59.3 \%-40 \%)$ from

425 the crescent side of fibers F4. It hence suggested that a round shape could provide a slower release. The time taken to release $60 \%, 80 \%$, and $95 \%$ of the loaded drug from fibers F3/F4 were 1.8/1.0, 4.1/3.0, and18.0/10.9 h (Fig. 5d). These values all indicated 
that the round shape of the EC-TAM compartment in F3 could provide a slower release profile than the crescent shape in F4.

To probe the drug release mechanisms from fibers F2 and the EC-TAM side of fibers F3 and F4, the Peppas equation (Peppas, 1985) (2) was used to analyze the experimental data.

$$
\mathrm{M}_{\mathrm{t}} / \mathrm{M}_{\infty}=\mathrm{kt}^{\mathrm{n}} \quad \text { (Eq. 2) }
$$

Where $M_{t} / M_{\infty}$ is the accumulative drug released fraction at time $t, t$ is the release 435 time, $\mathrm{k}$ is the release rate constant, and $\mathrm{n}$ is the diffusion index of the drug release. The curves were fitted as $F 2$ : $M_{t} / M_{\infty}=0.34 t^{0.38}\left(R^{2}=0.9857\right)$, the round side of fibers $F 3$ : $M_{t} / M_{\infty}=0.22 t^{0.38} \quad\left(R^{2}=0.9517\right)$, the crescent side of fibers $F 4: M_{t} / M_{\infty}=0.31 t^{0.24}$ $\left(\mathrm{R}^{2}=0.9849\right)$, respectively. The value of the diffusion index $\mathrm{n}$ for $\mathrm{F} 2$ was smaller than the critical value of 0.45 , suggested that the fibers F2 release the drug through a typical

440 Fickian diffusion mechanism. And the fitting results showed that the $n$ values for the ECTAM side of fibers F3 and F4 were 0.38 and 0.24 , respectively. They both are smaller than 0.45 , suggesting that the drug was released still through the typical Fickian diffusion mechanism. It indicated that the shape of EC-TAM side had no influence on the drug diffusion release mechanism. Still, it generated considerable effects on the drug release

445 behaviors and general release profiles through a special shape-performance relationship.

Polymers have long been vital in pharmaceutics. Most traditional dosage forms rely solely on the polymer properties to manipulate the drug release. In the new era of nanomedicine, however, complex nanostructures can be used to develop novel advanced 
DDSs. Here, as shown in Fig. 5e, Janus structures containing soluble PVP and insoluble

450 EC can integrate the functional performance of monolithic PVP and EC nanofibers to yield a biphasic drug release profile.

However, the core-shell nanofibers, regardless of the core or shell components, often have an inevitable round shape on their cross-sections. Here, based on the Janus nanostructures, the two sides' shape can be facilely manipulated. In the present work, the

455 round and crescent shape are combined as the two sides of the electrospun Janus fibers. For the fast release of drug from soluble polymer, the crescent shape has its advantage over the round shape. While for drug sustained release through a diffusion mechanism, the round shape is better than the crescent shape. It is because that the round shape has the smallest surface area in all of shapes, and the drug molecules loaded in the drug-

460 polymer composite always take the shortest way to diffuse into the dissolution medium. For example, a route through "A" is an easier route possibly for drug molecule release than the "B" route in the crescent shape in Fig. 5e. Thus, based on the structureperformance relationship, the Janus fibers F3 combines advantageous crescent PVPTAM side and round EC-TAM side, and correspondingly, they exhibited a better shape465 performance relationship than F4.

\section{Conclusions}

Two sets of Janus fibers have been prepared in this work, containing tamoxifen (TAM) as a pharmaceutical active ingredient, and with polyvinylpyrrolidone (PVP) and ethyl cellulose (EC) being the polymer matrices for the two sides. The fibers had linear 
and cylindrical morphologies with smooth surfaces and a Janus structure, as demonstrated by SEM and TEM. The two sides of the fiber have different shapes, with one being crescent and the other round. XRD and DSC results verified that the fibers comprised amorphous solid dispersions of the drug in the polymers, while FTIR spectroscopy indicated that TAM has good compatibility with both polymers. The shapes

475 of the sides had a distinct effect on functional performance. The F3 fibers, composed of a crescent PVP-TAM side and a round EC-TAM side, were able to provide a faster initial release and then a slower sustained release than the F4 system (consisting of round PVPTAM side and crescent EC-TAM side). Composition, structure, and shape are clearly all critical elements for designing functional nanomaterials. The protocols reported here

480 provide an example of how to combine these elements to develop advanced medical nanomaterials with precisely tailored functional performance.

\section{CRediT authorship contribution statement}

Xiaolu Zheng: Data curation, Investigation, Writing-original draft. Shixiong Kang: Data curation, Writing-original draft. Ke Wang: Investigation, Resources. Yaoyao Yang: 485 Methodology. Fuxian Wan: Writing - review \& editing. Deng-Guang Yu: Conceptualization, Funding acquisition, Project administration, Supervision, Writingreview \& editing. Gareth R. Williams: Conceptualization, Writing-review \& editing. Sim-Wan Annie Bligh: Conceptualization, Funding acquisition, Project administration, Supervision.

\section{Declaration of Competing Interest}

The authors declare that they have no known competing financial interests or personal relationships that could have appeared to influence the work reported in this paper.

\section{Acknowledgement}


This work was financially supported by the Shanghai Natural Science Foundation (No. 20ZR1439000) and the National Natural Science Foundation of China (No. 51803121).

\section{Appendix A. Supplementary material}

500 Supplementary data to this article can be found online at https:// doi.org/10.1016/j.ijpharm.2021.

\section{References:}

Agarwal, S., Wendorff, J.H., Greiner, A., 2008. Use of electrospinning technique for biomedical applications. Polymer 49, 5603-5621.

505 Aidana, Y.; Wang, Y.B.; Li, J.; Chang, S.Y.; Wang, K.; Yu, D.G., 2021. Fast dissolution electrospun medicated nanofifibers for effective delivery of poorly water soluble drugs. Curr. Drug Deliv. 18, 4-22.

Bai, Y.; Wang, D.; Zhang, Z.; Pan, J.; Cui, Z.; Yu, D.G.; Annie Bligh, S.W., 2021. Testing of fast dissolution of ibuprofen from its electrospun hydrophilic polymer nanocomposites. Polym. Test. 93, 106872.

Burstein, H.J., Lacchetti, C., Anderson, H., Buchholz, T.A., Davidson, N.E., Gelmon, K.E., Giordano, S.H., Hudis, C.A., Solky, A.J., Stearns, V., Winer, E.P., Griggs, J.J., 2016. Adjuvant endocrine therapy for women with hormone acceptor-positive breast cancer: American Society of Clinical Oncology Clinical Practice Guideline Update on Ovarian Suppression. J. Clin. Oncol. 34, 1689-1701.

Chang, S., Wang, M., Zhang, F., Liu, Y., Liu, X., Yu, D.-G., Shen, H., 2020. Sheathseparate-core nanocomposites fabricated using a trifluid electrospinning. Mater. Des. $192,108782$.

Chen, X.-Q., Ziemba, T., Huang, C., Chang, M., Xu, C., Qiao, J.X., Wang, T.C., Finlay, H.J., Salvati, M.E., Adam, L.P., Gudmundsson, O., Hageman, M.J., 2018. Oral Delivery of Highly Lipophilic, Poorly water-soluble drugs: Self-emulsifying drug delivery systems to improve oral absorption and enable high-dose toxicology studies of a cholesteryl ester transfer protein inhibitor in preclinical species. J. Pharm. Sci. 107, 1352-1360.

525 Cheng, G., Yin, C., Tu, H., Jiang, S., Wang, Q., Zhou, X., Xing, X., Xie, C., Shi, X., Du, Y., Deng, H., Li, Z., 2019. Controlled co-delivery of growth factors through layerby-layer assembly of core-shell nanofibers for improving bone regeneration. ACS Nano 13, 6372-6382. 
Démuth, B., Nagy, Z.K., Balogh, A., Vigh, T., Marosi, G., Verreck, G., Van Assche, I., Brewster, M.E., 2015. Downstream processing of polymer-based amorphous solid dispersions to generate tablet formulations. Int. J. Pharm. 486, 268-286.

Ding, Y.; Dou, C.; Chang, S.; Xie, Z.; Yu, D.G.; Liu, Y.; Shao, J., 2020. Core-shell Eudragit S100 nanofibers prepared via triaxial electrospinning to provide a colontargeted extended drug release. Polymers 12, 2034.

535 Du, X., Liu, Y., Wang, J., Niu, H., Yuan, Z., Zhao, S., Zhang, X., Cao, R., Yin, Y., Li, N., Zhang, C., Xing, Y., Xu, W., Li, C., 2018. Improved triboelectric nanogenerator output performance through polymer nanocomposites filled with core-shellstructured particles. ACS Appl. Mater. Interfaces 10, 25683-25688.

Duan, G., Greiner, A., 2019. Air-blowing-assisted coaxial electrospinning toward high productivity of core/sheath and hollow fibers. Macromol. Mater. Eng. 304, 1800669.

Grossen, P., Witzigmann, D., Sieber, S., Huwyler, J., 2017. PEG-PCL-based nanomedicines: A biodegradable drug delivery system and its application. J. Control. Release 260, 46-60.

Han, B., Xia, W., Liu, K., Tian, F., Chen, Y., Wang, X., Liang, F., Yang, Z., 2018. Janus Nanoparticles for Improved Dentin Bonding. ACS Appl. Mater. Interfaces 10, 8519-8526.

Hou, J., Yang, J., Zheng, X., Wang, M., Liu, Y., Yu, D.-G., 2020. A nanofiber-based drug depot with high drug loading for sustained release. Int. J. Pharm. 583, 119397.

Ibrahim, A.H., Smatt, J.-H., Govardhanam, N.P., Ibrahim, H.M., Ismael, H.R., Afouna, M.I., Samy, A.M., Rosenholm, J.M., 2020. Formulation and optimization of drugloaded mesoporous silica nanoparticle-based tablets to improve the dissolution rate of the poorly water-soluble drug silymarin. Eur. J. Pharm. Sci. 142, 105103.

Isaacoff, B.P., Brown, K.A., 2017. Progress in top-down control of bottom-up assembly. Nano Lett. 17, 6508-6510.

555 Jiang, S., Cheong, J.Y., Nam, J.S., Kim, I.-D., Agarwal, S., Greiner, A., 2020. Highdensity fibrous polyimide sponges with superior mechanical and thermal properties. ACS Appl. Mater. Interfaces 12, 19006-19014.

Jiang, S., Uch, B., Agarwal, S., Greiner, A., 2017. Ultralight, thermally insulating, compressible polyimide fiber assembled sponges. ACS Appl. Mater. Interfaces 9, 32308-32315.

Jordan, V.C., 2017. Endoxifen: The end, or are we at the beginning? J. Clin. Oncol. 35, 3378-3379. 
Kang, S.; Hou, S.; Chen, X.; Yu, D.G.; Wang, L.; Li, X.; Williams, G.R., 2021. Energysaving electrospinning with a concentric Teflon-core rod spinneret to create medicated nanofibers. Polymers 12, 2421.

Kim, B.Y.S., Rutka, J.T., Chan, W.C.W., 2010. Nanomedicine. N. Engl. J. Med. 363, 2434-2443.

Labbaf, S., Ghanbar, H., Stride, E., Edirisinghe, M., 2014. Preparation of multilayered polymeric structures using a novel four-needle coaxial electrohydrodynamic device. Macromol. Rapid Commun. 35, 618-623.

Li, C., Yin, Y., Wang, B., Zhou, T., Wang, J., Luo, J., Tang, W., Cao, R., Yuan, Z., Li, N., Du, X., Wang, C., Zhao, S., Liu, Y., Wang, Z.L., 2017. Self-powered electrospinning system driven by a triboelectric nanogenerator. ACS Nano 11, 10439-10445.

575 Li, M., Ai, M., Yang, Y., Yao, X., Zhou, Z., Wang, H., Li, C., Xu, K., 2020. Silk-coated dexamethasone non-spherical microcrystals for local drug delivery to inner ear. Eur. J. Pharm. Sci. 150, 105336.

Liao, X., Dulle, M., de Souza e Silva, J.M., Wehrspohn, R.B., Agarwal, S., Förster, S., Hou, H., Smith, P., Greiner, A., 2019. High strength in combination with high toughness in robust and sustainable polymeric materials. Science 366, 1376.

Liao, X., Ye, W., Chen, L., Jiang, S., Wang, G., Zhang, L., Hou, H., 2017. Flexible hdC$\mathrm{G}$ reinforced polyimide composites with high dielectric permittivity. Compos. Part A: Appl. S. 101, 50-58.

Mitragotri, S., Lahann, J., 2009. Physical approaches to biomaterial design. Nat. Mater. $8,15-23$.

Moghe, A.K., Gupta, B.S., 2008. Co-axial Electrospinning for nanofiber structures: Preparation and applications. Polym. Rev. 48, 353-377.

Nagy, Z.K., Balogh, A., Démuth, B., Pataki, H., Vigh, T., Szabó, B., Molnár, K., Schmidt, B.T., Horák, P., Marosi, G., Verreck, G., Van Assche, I., Brewster, M.E., 2015. High speed electrospinning for scaled-up production of amorphous solid dispersion of itraconazole. Int. J. Pharm. 480, 137-142.

Peppas, N.A., 1985. Analysis of Fickian and non-Fickian drug release from polymers. Pharm. Acta Helvet. 60, 110-111.

Ramzy, L., Nasr, M., Metwally, A.A., Awad, G.A.S., 2017. Cancer nanotheranostics: A review of the role of conjugated ligands for overexpressed receptors. Eur. J. Pharm. Sci. 104, 273-292. 
Santos, R.S., Figueiredo, C., Azevedo, N.F., Braeckmans, K., De Smedt, S.C., 2018. Nanomaterials and molecular transporters to overcome the bacterial envelope barrier: Towards advanced delivery of antibiotics. Adv. Drug Deliver. Rev. 136$137,28-48$.

Song, Y., Zhou, J., Fan, J.-B., Zhai, W., Meng, J., Wang, S., 2018. Hydrophilic/oleophilic magnetic Janus particles for the rapid and efficient oil-water separation. Adv. Funct. Mater. 28, 1802493.

Stearns, V., 2018. TAILORing adjuvant systemic therapy for breast cancer. N. Engl. J. 605 Med. 379, 191-192.

Viviane, A.G., Ligia, N.M.R., Giovana Radomille, T., Michelle, F.-M., Eneida de, P., Marcelo Bispo de, J., 2017. Current challenges and future of lipid nanoparticles formulations for topical drug application to oral mucosa, skin, and eye. Curr. Pharm. Des. 23, 6659-6675.

610 Wang, K., Liu, X. K., Chen,X. H., Yu, D.G., Yang, Y.Y., Liu, P., 2018. Electrospun hydrophilic Janus nanocomposites for the rapid onset of therapeutic action of helicid. ACS Appl. Mater. Interfaces 10, 2859-2867.

Wang, M.; Hou, J.; Yu, D.G.; Li, S.; Zhu, J.; Chen, Z., 2020b. Electrospun tri-layer nanodepots for sustained release of acyclovir. J. Alloy. Compd. 846, 156471.

615 Wang, M.; Li, D.; Li, J.; Li, S.; Chen, Z.; Yu, D.G.; Liu, Z.; Guo, J.Z., 2020c. Electrospun Janus zein-PVP nanofibers provide a two-stage controlled release of poorly watersoluble drugs. Mater. Des. 196, 109075.

Wang, M., Yu, D.-G., Li, X., Williams, G.R., 2020a. The Development and bioapplications of multifluid electrospinning. Mater. Highlights 1-2, 1-13.

620 Webster, T.J., 2006. Editorial: Nanomedicine: what's in a definition? Int. J. Nanomed. 1, 115-116.

Yao, K., Chen, J., Li, P., Duan, G., Hou, H., 2019. Robust strong electrospun polyimide composite nanofibers from a ternary polyamic acid blend. Compos. Commun. 15, 92-95.

625 Yoon, J., Yang, H.-S., Lee, B.-S., Yu, W.-R., 2018. Recent Progress in Coaxial Electrospinning: New Parameters, Various Structures, and Wide Applications. Adv. Mater. 30, 1704765.

Yu, D.G., 2021. Preface - Bettering drug delivery knowledge from pharmaceutical techniques and excipients. Curr. Drug Deliv. 18, 2-3. 
630 Zhang, S., Liu, H., Tang, N., Ge, J., Yu, J., Ding, B., 2019. Direct electronetting of highperformance membranes based on self-assembled 2D nanoarchitectured networks. Nat. Commun. 10, 1458.

Zhao, K.; Kang, S.X.; Yang, Y.Y.; Yu, D.G., 2021. Electrospun functional nanofiber membrane for antibiotic removal in water: Review. Polymers 13, 226.

635 Zheng, Y., Li, Z., Chen, H., Gao, Y., 2020. Nanoparticle-based drug delivery systems for controllable photodynamic cancer therapy. Eur. J. Pharm. Sci. 144, 105213.

Zhu, L.-F., Zheng, Y., Fan, J., Yao, Y., Ahmad, Z., Chang, M.-W., 2019. A novel coreshell nanofiber drug delivery system intended for the synergistic treatment of melanoma. Eur. J. Pharm. Sci. 137, 105002. 